\title{
Desempenho, características de carcaça e constituintes corporais de ovinos Santa Inês alimentados com farelo de girassol em substituição ao farelo de soja na dieta
}

\author{
Helder Louvandini ${ }^{1}$, Gustavo Aquino Nunes ${ }^{2}$, José Américo Soares Garcia ${ }^{1}$, Concepta \\ McManus ${ }^{1}$, Daniel Martins Costa $^{3}$, Samuel Ciminelli de Araújo ${ }^{3}$
}

\footnotetext{
1 FAV/UnB, Campus Universitário Darcy Ribeiro, Caixa Postal 04508, CEP: 70910-900, Brasília, DF.

2 Mestre pela Faculdade de Agronomia e Medicina Veterinária da Universidade de Brasília FAV/UnB.

${ }^{3}$ Graduando em Medicina Veterinária, bolsista PIBIC FAV/UnB.
}

RESUMO - Objetivou-se avaliar a inclusão do farelo de girassol na alimentação de ovinos Santa Inês. Dezoito cordeiros com 90 dias de idade, não-castrados, com $14 \pm 1,43 \mathrm{~kg}$ de PV inicial, foram distribuídos em um delineamento inteiramente casualizado e mantidos em confinamento durante 87 dias. Além do feno de coastcross (Cynodon dactylon) oferecido ad libitum, os cordeiros receberam $300 \mathrm{~g}$ de três diferentes concentrados contendo farelo de girassol em substituição ao farelo de soja. Os tratamentos utilizados foram: S - milho triturado + farelo de soja; SG - milho triturado $+50 \%$ de farelo de soja $+50 \%$ de farelo de girassol; e G - milho triturado $+100 \%$ de farelo de girassol. O controle do consumo de alimento foi feito três vezes por semana e a pesagem dos animais, uma vez. No abate, foram determinados o peso vivo (PV), o peso de carcaça quente (PC), o peso da meia-carcaça (PMC), o comprimento de carcaça, a espessura de gordura de cobertura, a pele (peso e expessura), o peso dos órgãos torácicos (pulmão, traquéia e coração) e abdominais (fígado e rins) e o peso dos cortes comerciais (pescoço, pernil, lombo, paleta, costela e costela/fralda). O ganho de peso médio diário e o ganho de peso total foram melhores nos animais alimentados com o concentrado composto de milho e farelo de soja. O consumo de MS por peso metabólico nesses animais (S) foi menor em relação ao obtido com o concentrado contendo $50 \%$ de girassol (SG), mas semelhante ao encontrado com $100 \%$ de farelo de girassol (G) $\mathrm{Na}$ avaliação das características de carcaça, os animais do grupo S apresentaram PMC, lombo e costela/fralda superiores aos dos grupos SG e G. A substituição do farelo de soja por farelo de girassol em até 50\% no concentrado promoveu menor desempenho e características de carcaça inferiores em ovinos em terminação.

Palavras-chave: confinamento, nutrição, terminação

\section{Performance, carcass characteristics and body measurements of Santa Inês sheep fed diets with different proportions of sunflower meal and soybean meal}

\begin{abstract}
The objective of this trial was to evaluate the effect of replacing soybean meal with sunflower meal on carcass traits and body measurements of Santa Inês lambs. Eighteen intact lambs averaging 90 days of age and $14 \pm 1.43 \mathrm{~kg}$ of body weight (BW) at the beginning of the study were assigned to a completely randomized design and confined during 87 days. Animals were fed coastcross (Cynodon dactylon) hay ad libitum plus $300 \mathrm{~g}$ of one of the following three concentrate mixtures: ground corn $+100 \%$ soybean meal $(\mathrm{SB})$, ground corn $+50 \%$ soybean meal $+50 \%$ sunflower meal (SBSF), or ground corn $+100 \%$ sunflower meal (SF). Feed intake was measured three times a week while body weight once a week. At slaughter the following measurements were taken: $\mathrm{BW}$, hot carcass and half carcass weights, carcass length, carcass fat cover, skin weight and thickness, thoracic (lung, trachea and heart) and abdominal (liver and kidneys) organ weights, and retail cut weights (neck, ham, loin, shoulder, rib and rib/belly). The average daily weight gain and total weight gain were greater on animals fed SB than on those fed SBSF and SF. However, DM intake expressed as metabolic weight was lower on SB diet compared to SBSF but similar to SF diet. Weights of half-carcass as well as loin and rib/belly weights were all higher on SB than on SBSF and SF diets. Replacing SB with SBSF decreased production and carcass characteristics in feedlot finishing lambs.
\end{abstract}

Key Words: confinement, nutrition, finishing

\section{Introdução}

Sabe-se que a nutrição é essencial para o sistema produtivo e que o farelo de girassol surge como alternativa alimentar para a criação de ruminantes. O farelo de girassol é um subproduto da indústria de óleos vegetais, resultante da moagem de sementes de girassol no processo industrial de extração do óleo, podendo ou não conter casca e apre- 
sentando grande variação na sua composição em proteína bruta, que oscila entre 28 e 44\% (Michael \& Sunde, 1985). A porcentagem de fibra bruta pode variar de 14 a $20,6 \%$ (Green \& Kiener, 1989) de acordo com a quantidade de casca presente no farelo. A grande variação na quantidade de fibra bruta pode levar a diferenças nos valores energéticos. Michael \& Sunde (1985) determinaram para o farelo de girassol valores de $1.907 \mathrm{kcal} \mathrm{EM} / \mathrm{kg}$.

Garcia et al. (2005), em experimento com bovinos holandeses em fase de crescimento, forneceram níveis de 0, 15, 30 e $45 \%$ de farelo de girassol nos concentrados e não observaram diferenças significativas para o consumo $(\mathrm{em} \mathrm{kg} / \mathrm{dia}$ e por peso metabólico) de MS, PB, EE, extrato não nitrogenado e cinzas. Entretanto, foram encontrados resultados significativos e aumento linear do ganho de peso vivo com o acréscimo de farelo de girassol na dieta. Esses autores concluíram que a inclusão de até $45 \%$ de farelo de girassol na dieta de bovinos leiteiros em fase de crescimento substituiu com eficiência o farelo de soja.

Além do ganho de peso, a qualidade de carcaça é importante na produção de carne. Portanto, estudos avaliando as características de carcaça devem ser associados a estudos de desempenho visando à seleção dos melhores animais para corte. As medidas realizadas na carcaça são fundamentais, pois permitem comparações entre tipos raciais, pesos e idades de abate, sistemas de alimentação e, ainda, o estabelecimento de correlações com outras medidas, como os componentes não-carcaça ou com os tecidos constituintes da carcaça, possibilitando a estimação de suas características físicas e evitando o oneroso processo de dissecação de carcaça (Silva et al., 1999).

Tendo em vista a possibilidade de oferta de diferentes tipos de farelos de girassol pela indústria e a escassez de dados sobre o assunto, este trabalho foi realizado com o objetivo de avaliar a utilização do farelo de girassol em substituição total e parcial ao farelo de soja na formulação do concentrado e seu efeito sobre o desempenho, as características de carcaça e os componentes não-carcaça de cordeiros Santa Inês terminados em confinamento.

\section{Material e Métodos}

Foram utilizados 18 cordeiros Santa Inês, não-castrados, com aproximadamente três meses de idade e $14 \pm 1,43 \mathrm{~kg}$ de $\mathrm{PV}$, confinados individualmente em baias com $1 \mathrm{~m}^{2}$ $(0,8 \mathrm{~m} \mathrm{x} \mathrm{1,2} \mathrm{m),} \mathrm{com} \mathrm{laterais} \mathrm{teladas} \mathrm{e} \mathrm{piso} \mathrm{ripado.} \mathrm{A} \mathrm{dieta} \mathrm{foi}$ composta de feno de coastcross (Cynodon dactylon) fornecido ad libitum, considerando uma sobra de $10 \%$ e $300 \mathrm{~g}$ de três misturas diferentes de concentrado (isoenergéticas e isoprotéicas): S - milho triturado + farelo soja; SG - milho triturado $+50 \%$ de farelo de soja $+50 \%$ de farelo de girassol; e $\mathrm{G}-$ milho triturado $+100 \%$ de farelo de girassol. Foi oferecida suplementação mineral (fosfato bicálcico), com $3,0 \mathrm{~g}$ de fósforo/animal/dia e mistura mineral de $9 \mathrm{~g} / \mathrm{animal} /$ dia (Kl - 0,009; CoSo4 - 0,0008; CuSO4 - 0,03; MgO - 1,61; $\mathrm{NaCl}-3,0 ; \mathrm{ZnSO} 4-0,32 ; \mathrm{MnSO} 4-0,148 ; \mathrm{FeSO} 4-0,457 ; \mathrm{S}$ - 4,0 g/dia). Na Tabela 1 constam os resultados das análises bromatológicas do feno de coastcross e dos concentrados utilizados na alimentação dos animais.

Antes do início do experimento, os animais foram vermifugados para controle parasitário e receberam aplicação de complexo vitamínico ADE via subcutânea. Depois de sete dias de adaptação, iniciou-se a coleta dos dados por 87 dias, efetuando-se o controle da ingestão de alimentos, por meio de pesagem (três vezes por semana) da quantidade fornecida e da respectiva sobra no cocho, e o acompanhamento do ganho de peso dos animais, por meio de pesagem semanal. Antes do abate, os animais foram pesados após jejum de sólidos (16 horas) e de líquidos (6 horas) para determinação do peso final. $\mathrm{O}$ abate foi realizado por atordoamento e degola em um abatedouro comercial. Após a sangria e a retirada da pele, efetuou-se uma abertura ao longo de toda a extensão da linha mediana ventral do abdômen para retirada das vísceras.

Em seguida, procedeu-se à retirada da cabeça e, mediante corte longitudinal da carcaça, obtiveram-se duas metades aproximadamente simétricas, de modo que, no antímero esquerdo, foram realizados os seguintes cortes comerciais pescoço, pernil, lombo, paleta, costela e costela/fralda e, no músculo Longissimus dorsi, foi determinada a área de olhode-lombo na altura da $12^{\mathrm{a}}$ costela, com um corte transversal

Tabela 1 - Composição química (\%MS) do feno de coastcross (Cynodon dactylon) e dos concentrados utilizados na alimentação dos animais

Table 1 - Chemical composition (\%DM) of coast-cross hay (Cynodon dactylon) and concentrates

\begin{tabular}{ccc}
\hline Item & Feno & Concentrado \\
Hay & Concentrate
\end{tabular}

\begin{tabular}{lcccc} 
& & $\begin{array}{c}\text { Farelo de } \\
\text { girassol } \\
\text { Sunflower } \\
\text { meal }\end{array}$ & $\begin{array}{c}\text { Farelo de soja/ } \\
\text { Farelo de girassol } \\
\text { Soybean meal }+ \\
\text { Sunflower meal }\end{array}$ & $\begin{array}{c}\text { Farelo de } \\
\text { soja } \\
\text { Soybean } \\
\text { meal }\end{array}$ \\
\hline MS (DM) & 89,92 & 89,65 & 89,36 & 89,45 \\
PB $(C P)$ & 3,31 & 14,43 & 14,14 & 14,95 \\
EE & 0,81 & 2,73 & 2,39 & 2,36 \\
FDN (NDF) & 83,02 & 23,30 & 20,39 & 15,32 \\
FDA (ADF) & 47,27 & 7,92 & 5,94 & 3,91 \\
Cinzas $(A s h)$ & 2,6 & 7,3 & 6,74 & 7,95 \\
\hline
\end{tabular}


e um desenho do músculo em papel vegetal, possibilitando a utilização de gabaritos quadriculados, cada um com $1 \mathrm{~cm}^{2}$.

As características de carcaça foram avaliadas conforme procedimentos adaptados de Osório et al. (1998), efetuando-se a pesagem e avaliação para determinação do peso de carcaça quente, da cobertura de gordura (1 para magra a 5 muito gorda), do comprimento de carcaça, do peso e da espessura da pele, do peso dos órgãos torácicos (pulmão, traquéia e coração) e abdominais (fígado e rins) e da circunferência e do peso escrotal.

Para determinação da composição química da 12 a costela (como parâmetro da avaliação da composição da carcaça), foi realizada a dissecação, pela separação de seus componentes (gordura, músculo e osso) e respectiva pesagem. Em seguida, os componentes teciduais das costelas foram novamente agrupados (gordura + músculo + osso), moídos e congelados a $-20^{\circ} \mathrm{C}$ para liofilização e determinação do teor de MS. Sequiencialmente, as amostras foram trituradas novamente para análise química e determinação das porcentagens de $\mathrm{PB}, \mathrm{EE}$, cinzas e $\mathrm{MS}$ corrigida a $105^{\circ} \mathrm{C}$, segundo AOAC (1995).

O delineamento experimental foi o inteiramente ao acaso, com três tratamentos e seis repetições. Utilizou-se o programa estatístico Statistical Analysis System (SAS, 1996) para análise de variância dos parâmetros testados e das diferenças entre as médias, determinadas pelo teste Tukey a 5\% de significância.

\section{Resultados e Discussão}

O consumo é a função do animal que está diretamente associada ao peso vivo, ao nível de produção, à variação do peso vivo e ao estado fisiológico, além do tipo de alimento e das condições de alimentação (Mertens, 1983). Neste estudo, teve-se o cuidado de promover o consumo total dos concentrados das dietas em todos os tratamentos, portanto, a variação de consumo refere-se ao volumoso. O consumo de matéria seca por peso vivo (CMS/PV) não diferiu $(\mathrm{P}>0,05)$ entre os animais alimentados com as diferentes dietas. Logo, a substituição de farelo de soja por farelo de girassol não proporcionou redução no CMS quando expresso em kg de peso vivo (Tabela 2). O consumo de MS expresso em g/ $\mathrm{kg} \mathrm{PV}^{0,75}$ foi maior $(\mathrm{P}<0,05)$ nos animais alimentados com a dieta $\mathrm{SG}$ em comparação à dieta $\mathrm{S}$. A substituição do farelo de soja por farelo de girassol no concentrado ocasionou aumento no consumo de volumoso, todavia, esse aumento não proporcionou melhor desempenho aos animais.

O consumo diário de alimento encontrado por Medeiros et al. (2003) em experimento com cordeiros Santa Inês em confinamento em fases de recria e terminação recebendo feno de capim-elefante como volumoso foram 1,20; 1,20 ; 1,$18 ;$ e $1,11 \mathrm{~kg} /$ dia, respectivamente, para os níveis de 0,33 , 66 e $100 \%$ de substituição do farelo de soja por farelo de girassol. O consumo de matéria seca (CMS) observado por esses autores foi praticamente inalterado entre os níveis de 0 e $66 \%$ de farelo de girassol, sendo observadas ingestões de 1,20 e $1,18 \mathrm{~kg} / \mathrm{dia}$, respectivamente. No nível de $100 \%$ de substituição, no entanto, houve redução no teor energético ingerido pelos animais, como resultado da redução no consumo de MS e da concentração energética das dietas.

No início do experimento, não foi verificada diferença significativa $(\mathrm{P}>0,05)$ no peso vivo dos cordeiros entre os tratamentos, porém, ao término do trabalho, verificou-se uma tendência de os animais alimentados somente com soja serem mais pesados que aqueles alimentados com o concentrado contendo exclusivamente girassol $(\mathrm{P}=0,0910)$. Entretanto, quando avaliados o ganho de peso diário e o ganho de peso total, a dieta diferiu significativamente $(\mathrm{P}<0,05)$ das demais. Em trabalho conduzido por Medeiros et al. (2003), os ganhos de peso médios diários foram de 190, 170,150 e $100 \mathrm{~g}$, respectivamente, para $0,33,66$ e $100 \%$ de níveis de substituição do farelo de soja por farelo de girassol, o que está de acordo com os resultados obtidos nesta pesquisa, ou seja, à medida que se aumentou a

Tabela 2 - Desempenho (valores médios) de ovinos alimentados com diferentes níveis de farelo de girassol em substituição ao farelo de soja no concentrado

Table 2 - Performance of lambs fed diets with different proportions of soybean meal and sunflower meal

\begin{tabular}{|c|c|c|c|c|}
\hline Item & $\begin{array}{l}\text { Farelo de girassol } \\
\text { Sunflower meal }\end{array}$ & $\begin{array}{l}\text { Farelo de soja/Farelo de girassol } \\
\text { Soybean meal }+ \text { Sunflower meal }\end{array}$ & $\begin{array}{l}\text { Farelo de soja } \\
\text { Soybean meal }\end{array}$ & $\begin{array}{l}\text { Erro-padrão } \\
\text { Standard error }\end{array}$ \\
\hline Peso vivo inicial $(\mathrm{kg})$ (Initial weight, $\mathrm{kg}$ ) & 17,0 & 16,25 & 15,58 & 1,43 \\
\hline Peso vivo final $(\mathrm{kg})$ (Final weight, $\mathrm{kg}$ ) & 24,66 & 25,08 & 27,75 & 1,21 \\
\hline Ganho de peso diário $(\mathrm{g})$ (Daily weight gain, $g$ ) & $88,12^{\mathrm{b}}$ & $101,53^{\mathrm{b}}$ & $139,84^{\mathrm{a}}$ & 9,91 \\
\hline Ganho de peso total $(\mathrm{kg})$ (Total weight gain, $\mathrm{kg}$ ) & $7,66^{\mathrm{b}}$ & $8,83^{\mathrm{b}}$ & $12,16^{\mathrm{a}}$ & 0,86 \\
\hline Consumo de MS/PV $(\mathrm{g} / \mathrm{kg})$ (DM intake/BW, $g / \mathrm{kg}$ ) & 40,58 & 40,92 & 36,03 & 1,84 \\
\hline Consumo de MS/PV ${ }^{0,75}(\mathrm{~g} / \mathrm{kg})\left(\right.$ DM intake/BW $\left.W^{0.75}\right)$ & $87,68^{\mathrm{ab}}$ & $88,32^{\mathrm{a}}$ & $79,27^{\mathrm{b}}$ & 3,07 \\
\hline Conversão alimentar (Feed conversion) & $10,86^{\mathrm{b}}$ & $9,11^{\mathrm{a}}$ & $6,58^{a}$ & 0,93 \\
\hline
\end{tabular}

a,b Médias seguidas de letras diferentes na mesma linha diferem $(P<0,05)$ pelo teste Tukey.

a,b Means followed by different letters in the same row differ $(P<0.05)$ by Tukey test. 
quantidade de farelo de girassol na dieta, o ganho de peso diário diminuiu.

O consumo e o ganho de peso refletiram na conversão alimentar, que, nos animais alimentados com as dietas $\mathrm{S}$ e $\mathrm{SG}$, foram semelhantes, porém, superiores aos obtidos nos grupos $\mathrm{G}(\mathrm{P}<0,05)$, como resultado do menor consumo de MS por peso metabólico. Estes resultados foram inferiores aos encontrados por Medeiros et al. (2003), que obtiveram conversões alimentares de 6,26 e 7,12, respectivamente, nos níveis de 0 e $100 \%$ de substituição de farelo de soja por farelo de girassol.

Os animais alimentados com a da dieta $\mathrm{S}$ apresentaram valores de peso de carcaça superiores aos obtidos com a dieta $\mathrm{G}(\mathrm{P}<0,05)$, mas não diferiram significativamente em comparação aos da dieta SG (Tabela 3). O peso da meiacarcaça foi maior nos animais alimentados com a dieta $\mathrm{S}$ $(\mathrm{P}<0,05)$, provavelmente em decorrência da maior conversão alimentar e do ganho de peso dos cordeiros alimentados com a dieta $\mathrm{S}$.

Os resultados obtidos para o peso de carcaça nesta pesquisa foram semelhantes aos encontrados por Müller et al. (2003), ao confinarem 12 cordeiros cruza Ile de France $x$ Texel desmamados com 45 dias de idade e peso médio de 16 $\mathrm{kg}$ alimentados com silagem e concentrado à base de milho e farelo de soja. Apesar de o rendimento de carcaça não ter diferido significativamente $(\mathrm{P}>0,05)$ entre as dietas, os resultados obtidos para esta variável estão na faixa aceita para ovinos, de 40 a 50\% (Macedo et al., 1999). O comprimento de carcaça também não diferiu significativamente entre as dietas $(\mathrm{P}>0,05)$, embora o grupo $\mathrm{S}$ tenha apresentado valor numérico maior.

A proporção de gordura de cobertura foi numericamente maior nos animais alimentados com a dieta sem farelo de girassol $(3,5)$, no entanto, os valores não diferiram significativamente entre os tratamentos $(\mathrm{P}>0,05)$. A média para a cobertura de gordura foi de 2,99 , comprovando que o acabamento foi satisfatório nos animais dos três grupos.

A condição corporal determina a melhor época de abate para obtenção de carcaças com bom acabamento. As carcaças com pouca cobertura de gordura ou com cobertura de gordura não uniforme dessecam mais rapidamente no processo de armazenamento a frio, gerando depreciação do produto (Bueno et al., 2000). O excesso de gordura também pode ser fator restritivo na escolha pelo consumidor. Ressalta-se que esta é uma medida subjetiva que depende muito da pessoa que está realizando as medidas, o que, às vezes, torna complicada a comparação entre trabalhos.

Os valores para proporção de pernil na meia-carcaça obtidos com a dieta $\mathrm{S}$ foram inferiores $(\mathrm{P}<0,05)$ aos encontrados nos demais tratamentos. Do mesmo modo, os resultados da proporção de paleta na meia-carcaça nos animais alimentados com dieta $\mathrm{S}$ foram inferiores aos obtidos com a dieta $\mathrm{SG}(\mathrm{P}<0,05)$, porém, semelhantes aos da dieta $\mathrm{G}$. Assim, os cordeiros menos pesados ( $\mathrm{SG} \mathrm{e} \mathrm{G}$ ) apresentaram

Tabela 3 - Características de carcaça e de ovinos alimentados com diferentes níveis de farelo de girassol em substituição ao farelo de soja no concentrado

Table 3 - $\quad$ Carcass characteristics of lambs fed diets with different proportions of soybean meal and sunflower meal

\begin{tabular}{|c|c|c|c|c|}
\hline $\begin{array}{l}\text { Característica } \\
\text { Characteristic }\end{array}$ & $\begin{array}{l}\text { Farelo de girassol } \\
\text { Sunflower meal }\end{array}$ & $\begin{array}{l}\text { Farelo de soja/Farelo de girassol } \\
\text { Soybean meal }+ \text { Sunflower meal }\end{array}$ & $\begin{array}{l}\text { Farelo de soja } \\
\text { Soybean meal }\end{array}$ & $\begin{array}{l}\text { Erro-padrão } \\
\text { Standard error }\end{array}$ \\
\hline Peso carcaça $(\mathrm{kg})$ (Carcass weight, $\mathrm{kg}$ ) & $10,7^{\mathrm{b}}$ & $10,98^{\mathrm{ab}}$ & $12,2^{\mathrm{a}}$ & 0,503 \\
\hline Rendimento de carcaça (\%) (Carcass yield, \%) & 45,00 & 45,66 & 46,66 & 0,700 \\
\hline Peso da meia-carcaça ( $\mathrm{kg}$ ) (Half carcass weight, $\mathrm{kg}$ ) & $5,38^{b}$ & $5,45^{b}$ & $6,20^{\mathrm{a}}$ & 0,244 \\
\hline Comprimento de carcaça $(\mathrm{cm})$ (Carcass length, $\mathrm{cm})$ & 61,91 & 62,50 & 64,83 & 1,498 \\
\hline Gordura de cobertura ( 1 a 5) (Carcass fatness, 1 until 5) & 2,66 & 2,83 & 3,5 & 0,389 \\
\hline Pernil (Leg) & & & & \\
\hline$(\mathrm{kg})$ & 1,98 & 2,05 & 2,10 & 0,845 \\
\hline$(\%)$ & $36,83^{a}$ & $37,66^{\mathrm{a}}$ & $33,83^{b}$ & 0,86 \\
\hline \multicolumn{5}{|l|}{ Paleta (Shoulder) } \\
\hline$(\mathrm{kg})$ & 1,11 & 1,16 & 1,23 & 0,057 \\
\hline$(\%)$ & $20,66^{\mathrm{ab}}$ & $21,33^{\mathrm{a}}$ & $19,83^{b}$ & 0,51 \\
\hline \multicolumn{5}{|l|}{ Lombo (Back) } \\
\hline$(\mathrm{kg})$ & $0,48^{b}$ & $0,46^{b}$ & $0,61^{\mathrm{a}}$ & 0,045 \\
\hline$(\%)$ & 8,83 & 8,50 & 10,16 & 0,058 \\
\hline \multicolumn{5}{|l|}{ Costela (Rib) } \\
\hline$(\mathrm{kg})$ & $0,76^{a b}$ & $0,71^{\mathrm{b}}$ & $0,91^{\mathrm{a}}$ & 0,060 \\
\hline$(\%)$ & 14,50 & 13,33 & 14,50 & 0,81 \\
\hline \multicolumn{5}{|l|}{ Costela/Fralda (Rib/Belly) } \\
\hline$(\mathrm{kg})$ & $0,65^{b}$ & $0,68^{\mathrm{b}}$ & $0,81^{\mathrm{a}}$ & 0,035 \\
\hline$(\%)$ & 12,00 & 12,50 & 13,00 & 0,38 \\
\hline
\end{tabular}

a,b Médias seguidas por letras diferentes na mesma linha diferem $(P<0,05)$ pelo teste Tukey.

$a, b$ Means followed by different letters in the same row differ $(P<0.05)$ by Tukey test. 
maior proporção desses cortes nobres, à exceção do lombo, que foi maior nos animais alimentados com a dieta $S$ em relação às demais $(\mathrm{P}<0,05)$. Resultados semelhantes foram encontrados por Pires et al. (2006), que obtiveram 31,66; 19,53 e $17,09 \%$, respectivamente, para os rendimentos de pernil, paleta e costela de cordeiros com 12,66 kg de peso de carcaça. Os cordeiros alimentados com a dieta $\mathrm{S}$ apresentaram maior peso de costelas $(\mathrm{P}<0,05)$ e foram diferentes daqueles alimentados com a dieta $\mathrm{SG}$ e semelhantes aos da G. Diferenças significativas $(\mathrm{P}<0,05)$ foram constatadas também para costela/fralda nos animais alimentados com a dieta $\mathrm{S}$, os quais apresentaram maior peso desse corte em comparação aos cordeiros dos demais grupos.

Segundo Hammond (1966), à medida que o ovino cresce, ocorrem modificações em suas proporções corporais, verificando-se geralmente uma onda de crescimento que se inicia na cabeça e se estende ao longo do tronco (ondas primárias) e outras que se iniciam nas extremidades e ascendem pelo corpo, encontrando-se na região do lombo com a última costela "região de menor desenvolvimento" (ondas secundárias). Os valores de costela, costela/fralda, paleta e pernil obtidos neste trabalho confirmam essa afirmativa, isto é, os animais mais pesados apresentaram maior desenvolvimento da região do flanco em relação aos membros. Os mesmos resultados foram observados por Furusho-Garcia et al. (2004) em estudo com ovinos puros Santa Inês e seus cruzamentos com Ile de France, Texel e Bergamacia.

Os pesos e as proporções, em relação à meia-carcaça, da pele, do escroto, da circunferência escrotal e do peso dos órgãos abdominais não diferiram significativamente $(\mathrm{P}>0,05)$ entre as dietas (Tabela 4). Entretanto, a espessura de pele e o peso dos órgãos torácicos dos cordeiros alimentados com a dieta $\mathrm{G}$ foram maiores $(\mathrm{P}<0,05)$ em comparação aos valores obtidos com a dieta $\mathrm{SG}$, mas semelhantes $(\mathrm{P}>0,05)$ aos obtidos com a dieta $\mathrm{S}$. A proporção dos órgãos torácicos foi maior $(\mathrm{P}<0,05)$ nos cordeiros que receberam a dieta $\mathrm{G}$, enquanto a proporção dos órgãos abdominais foi maior $(\mathrm{P}<0,05)$ nos animais alimentados com a dieta $\mathrm{SG}$, porém semelhante $(\mathrm{P}>0,05)$ à encontrada nos animais alimentados com a dieta G. Essas diferenças estão associadas ao maior desenvolvimento corporal promovido pelas dietas SG e S, aumentando a estrutura de carcaça em detrimento dos órgãos torácicos e abdominais, resultados identificados também por Silva Sobrinho et al. (2003) para coração e pulmões. As vísceras apresentam crescimento mais precoce, portanto, tendem a diminuir com a evolução do peso corporal.

Órgãos e vísceras também possuem distintas velocidades de crescimento durante a vida do animal, quando comparados a outras partes do corpo, o pode estar relacionado à composição química dos alimentos, especialmente, ao teor de energia. Portanto, é necessária a valorização do animal como um todo. Além disso, o aproveitamento desses componentes não-carcaça pode ser uma nova fonte de renda para o mercado promissor da ovinocultura (Kamalzadeh et al., 1998).

Os resultados referentes à análise da $12^{\mathrm{a}}$ costela (peso e proporções de músculo, gordura, osso e composição química) e da área de olho-de-lombo dos ovinos alimentados com os diferentes concentrados encontram-se na Tabela 5.

Os resultados obtidos para peso da $12^{2}$ costela, músculo, gordura e osso nos animais alimentados com a dieta $\mathrm{S}$ foram superiores $(\mathrm{P}<0,05)$ aos daqueles que receberam a dieta $\mathrm{SG}$,

Tabela 4 - Valores médios para pesos e porcentagens dos componentes não-carcaça em relação à meia-carcaça de ovinos alimentados com diferentes níveis de farelo de girassol em substituição ao farelo de soja

Table 4 - Mean values for weights and proportions of noncarcass components relative to half-carcass weight of lambs fed diets with different proportions of soybean meal and sunflower meal

\begin{tabular}{|c|c|c|c|c|}
\hline $\begin{array}{l}\text { Componente } \\
\text { Component }\end{array}$ & $\begin{array}{l}\text { Farelo de girassol } \\
\text { Sunflower meal }\end{array}$ & $\begin{array}{l}\text { Farelo de soja/Farelo de girassol } \\
\text { Soybean meal }+ \text { Sunflower meal }\end{array}$ & $\begin{array}{l}\text { Farelo de soja } \\
\text { Soybean meal }\end{array}$ & $\begin{array}{l}\text { Erro-padrão } \\
\text { Standard error }\end{array}$ \\
\hline \multicolumn{5}{|l|}{ Pele (Skin) } \\
\hline$(\mathrm{kg})$ & 1,33 & 1,41 & 1,60 & 0,09 \\
\hline (\%) & 24,66 & 25,83 & 26,40 & 50,55 \\
\hline Espessura de pele $(\mathrm{mm})$ (Skin thickness, $\mathrm{mm}$ ) & $2,00^{\mathrm{a}}$ & $1,16^{\mathrm{b}}$ & $1,60^{\mathrm{ab}}$ & 0,256 \\
\hline \multicolumn{5}{|l|}{ Órgãos torácicos (Thoracic organs) } \\
\hline$(\mathrm{kg})$ & $0,56^{\mathrm{a}}$ & $0,46^{\mathrm{b}}$ & $0,55^{\mathrm{ab}}$ & 0,033 \\
\hline$(\%)$ & $10,60^{\mathrm{b}}$ & $8,50^{\mathrm{a}}$ & $8,75^{\mathrm{a}}$ & 0,60 \\
\hline \multicolumn{5}{|l|}{ Órgãos abdominais (Abdominal organs) } \\
\hline$(\mathrm{kg})$ & 0,62 & 0,66 & 0,65 & 0,038 \\
\hline$(\%)$ & $11,80^{\mathrm{ab}}$ & $12,50^{\mathrm{a}}$ & $10,50^{\mathrm{b}}$ & 0,56 \\
\hline \multicolumn{5}{|l|}{ Escroto (Scrotum) } \\
\hline$(\mathrm{kg})$ & 0,100 & 0,108 & 0,112 & 0,019 \\
\hline$(\%)$ & 1,88 & 1,96 & 1,81 & 0,34 \\
\hline Circunferência escrotal $(\mathrm{cm})$ (Scrotal circumference, $\mathrm{cm})$ & 22,91 & 22,50 & 22,80 & 0,960 \\
\hline
\end{tabular}

a,b Médias seguidas por letras diferentes na mesma linha diferem $(P<0,05)$ pelo teste Tukey.

$a, b$ Means followed by different letters in the same row differ $(P<0.05)$ by Tukey test. 
Tabela 5 - Valores médios para peso e porcentagens de músculo, gordura, osso, área de olho-de-lombo e composição química da 12a costela de ovinos alimentados com diferentes níveis de farelo de girassol em substituição ao farelo de soja

Table 5 - Mean values for weight and proportion of muscle, fat, bone, and loin eye are and chemical composition of the $12^{\text {th }}$ rib of lambs fed diets with different proportions of soybean meal and sunflower meal

\begin{tabular}{|c|c|c|c|c|}
\hline Item & $\begin{array}{l}\text { Farelo de girassol } \\
\text { Sunflower meal }\end{array}$ & $\begin{array}{l}\text { Farelo de soja/Farelo de girassol } \\
\text { Soybean meal }+ \text { Sunflower meal }\end{array}$ & $\begin{array}{l}\text { Farelo de soja } \\
\text { Soybean meal }\end{array}$ & $\begin{array}{l}\text { Erro-padrão } \\
\text { Standard error }\end{array}$ \\
\hline $\begin{array}{l}\text { Peso da } 12^{\mathrm{a}} \text { costela }(\mathrm{g})\left(12^{\text {th }} \text { rib weight, } g\right) \\
\text { Músculo (Muscle) }\end{array}$ & $69,18^{\mathrm{ab}}$ & $61,73^{\mathrm{b}}$ & $84,83^{\mathrm{a}}$ & 5,017 \\
\hline (g) & $38,10^{\mathrm{ab}}$ & $37,62^{b}$ & $46,47^{\mathrm{a}}$ & 2,565 \\
\hline$(\%)$ & 55,07 & 61,97 & 55,13 & 2,84 \\
\hline \multicolumn{5}{|l|}{ Gordura (Fat) } \\
\hline (g) & $9,76^{a b}$ & $7,54^{\mathrm{b}}$ & $12,37^{\mathrm{a}}$ & 1,508 \\
\hline$(\%)$ & 14,22 & 11,70 & 14,47 & 1,71 \\
\hline \multicolumn{5}{|l|}{ Osso (Bone) } \\
\hline$(\mathrm{g})$ & $18,47^{\mathrm{ab}}$ & $13,10^{\mathrm{b}}$ & $20,65^{\mathrm{a}}$ & 2,343 \\
\hline$(\%)$ & 26,86 & 20,87 & 23,85 & 2,22 \\
\hline Área de olho-de-lombo $\left(\mathrm{cm}^{2}\right)$ (Eye loin area, $\mathrm{cm}^{2}$ ) & 9,18 & 9,58 & 10,15 & 0,62 \\
\hline \multicolumn{5}{|l|}{$\begin{array}{l}\text { Composição química da } 12^{\mathrm{a}} \text { costela } \\
\text { Chemical composition of the } 12^{\text {th }} \mathrm{rib}\end{array}$} \\
\hline $\operatorname{MS}(\%)(D M)$ & 37,40 & 38,85 & 39,78 & 1,28 \\
\hline $\mathrm{PB}(\%)(C P)$ & 46,87 & 47,00 & 45,05 & 1,80 \\
\hline $\mathrm{EE}(\%)(E E)$ & 28,38 & 29,07 & 30,61 & 2,07 \\
\hline Cinzas $(\%)(A s h)$ & 16,11 & 14,88 & 15,15 & 1,14 \\
\hline
\end{tabular}

a,b Médias seguidas por letras diferentes na mesma linha diferem $(P<0,05)$ pelo teste Tukey.

$a, b$ Means followed by different letters in the same row differ $(P<0.05)$ by Tukey test.

porém, semelhantes aos da dieta G. As porcentagens desses componentes, no entanto, foram semelhantes entre as três dietas. O mesmo ocorreu com as porcentagens de MS, PB, EE e cinzas ( $P>0,05)$. Segundo Silva \& Pires (2000), em ovinos mestiços (Texel $\times$ Ideal) abatidos com 4,12 a 32,57 kg, o melhor equilíbrio nas características quantitativas da carcaça foi observado em animais abatidos com $28 \mathrm{~kg}$. De acordo com esses autores, a costela foi o corte que melhor expressou a relação osso:músculo:gordura na carcaça. Como as dietas foram isoenergéticas e isoprotéicas e diferiram apenas quanto à qualidade do ingrediente, no peso global, o desempenho foi melhor nos animais alimentados com a dieta contendo farelo de soja, no entanto, as proporções dos tecidos se mantiveram semelhantes.

A área de olho-de-lombo tem sido freqüentemente utilizada na predição da quantidade de músculo da carcaça, contudo, trabalhos têm demonstrado que esta variável deve ser utilizada com outras características para melhor avaliar a composição muscular da carcaça (Muller, 1980; Silva \& Pires, 2000). Neste trabalho, os cordeiros que receberam as dietas $\mathrm{S}$ e SG apresentaram valores numéricos superiores para esta variável, no entanto, essas diferenças não foram significativas $(\mathrm{P}>0,05)$, sendo observada uma associação ao peso corporal dos ovinos, confirmando tratar-se de um músculo de crescimento tardio. Os valores encontrados para área de olho-de-lombo neste estudo foram inferiores aos obtidos por Garcia et al. (2003) e Tonetto et al. (2004), que registraram, respectivamente, valores de 12,62 e 13,48 $\mathrm{cm}^{2}$, todavia, nos experimentos realizados por esses autores, foram utilizados animais mais pesados $(31 \mathrm{~kg})$, o que justifica essas diferenças.

\section{Conclusões}

A substituição de 100 e de $50 \%$ do farelo de soja pelo farelo de girassol no concentrado proporcionou menor ritmo de crescimento e características de carcaça inferiores em ovinos Santa Inês em terminação, comprovando que os níveis de substituição estudados não são indicados para a categoria animal avaliada.

\section{Literatura Citada}

ASSOCIATION OF OFFICIAL AGRICULTURAL CHEMISTS Official methods of analysis of the AOAC. 16.ed. Arlington: AOAC International, 1995. v.1, p.4-30.

BUENO, M.; CUNHA, E.A.; SANTOS, L.E. et al. Características de carcaças de cordeiros Suffolk abatidos em diferentes idades. Revista Brasileira de Zootecnia, v.29, n.6, p.1803-1810, 2000

FURUSHO-GARCIA, I.F.; PEREZ, J.R.O.; BONAGURIO, S. et al. Estudo dos cortes da carcaça de cordeiros Santa Inês puros e cruzas Santa Inês com Texel, Ile de France e Bergamacia. Revista Brasileira de Zootecnia, v.33, n.2, p.453-462, 2004.

GARCIA, J.A.S.; VIEIRA, P.F.; CECON, P.R et al. Ganho em peso e mensurações corporais em bovinos leiteiros em fase de crescimento alimentados com farelo de girassol. Ciência Animal Brasileira, no prelo, 2005.

GARCIA, C.A.; MONTEIRO, A.L.G.; COSTA, C. et al. Medidas objetivas e composição tecidual da carcaça de cordeiros alimentados com diferentes níveis de energia em creep feeding. Revista Brasileira de Zootecnia, v.32, n.6, p.1380-1390, 2003. 
GREEN, S.; KIENER, T. Digestibilities of nitrogen and amino acids in soybean, sunflower, meat and rapeseed meals measured with pigs and poultry. Animal Production, v.48, p.157-179, 1989.

HAMMOND, J. Principios de la explotación animal. Zaragoza: Acribia, 1966. 363p.

KAMALZADEH, A.; KOOPS, W.J.; van BRUCHEM et al. Feed quality restriction and compensatory growth in growing sheep: development of body organs. Small Ruminant Research, v.29, n.1, p.71-82, 1998.

MACEDO, F.O.F.; SIQUEIRA, E.R.; MARTINS, E.N. Desempenho de cordeiros Corriedale puros e mestiços, terminados em pastagem e em confinamento. Arquivo Brasileiro Medicina Veterinária Zootecnia, v.51, n.6, p.583-587, 1999.

MEDEIROS, O.N.; HONÓRIO, F.O.; LISBOA, O.C. et al. Desempenho de cordeiros Santa Inês alimentados com farelo de girassol. In: REUNIÃO ANUAL DA SOCIEDADE BRASILIERA DE ZOOTECNA, 40., 2003, Santa Maria. Anais... Santa Maria: Sociedade Brasileira de Zootecnia, 2003. (CD-ROM).

MERTENS, D.R. Using neutral detergent fibre to formulate dairy ration and estimative the net energy content of feeds. In: CORNELL NUTRIENT CONFERENCE, Ithaca, 1983. Proceedings... Ithaca: 1983. p.60-68.

MICHAEL, J.N.; SUNDE, M.L. Sunflower meals in pullet developer diets. Poultry Science, v.64, p.669-674, 1985.

MÜLLER, L. Normas para avaliação de carcaças e concurso de carcaças de novilhos. Santa Maria: Universidade Federal de Santa Maria, 1980. 31p.

MÜLLER, L.; PIRES, C.C.; SILVA, V.S. et al. Características da carcaça de cordeiros desmamados aos 45 dias em três tipos de confinamentos. In: REUNIÃO ANUAL DA SOCIEDADE BRASILIERA DE ZOOTECNA, 40., 2003, Santa Maria. Anais... Santa Maria: Sociedade Brasileira de Zootecnia, 2003. (CD-ROM).

OSÓRIO, J.C.S.; OSÓRIO, M.T.M.; JARDIM, P.O.C. et al. Métodos para avaliação da produção de carne ovina: in vivo, na carcaça e na carne. Pelotas: Universidade Federal de Pelotas, 1998. $107 p$.
PIRES, C.C.; GALVANI, D.B.; CARVALHO, S. et al. Características da carcaça de cordeiros alimentados contendo diferentes níveis de fibra em detergente neutro. Revista Brasileira de Zootecnia, v.35, n.5, p.2058-2065, 2006.

STATISTICAL ANALYSIS SYSTEM - SAS. User's guide. 5ed. Cary, 1996. 956p.

SILVA, L.F.; PIRES, C.C. Avaliações quantitativas e predição das proporções de osso, músculo e gordura da carcaça em ovinos. Revista Brasileira de Zootecnia, v.29, n.4, p.1253-1260, 2000.

SILVA, L.F.; PIRES, C.C.; PEIXOTO, L.O. et al. Constituintes corporais de cordeiros abatidos com diferentes pesos. In: REUNIÃO ANUAL DA SOCIEDADE BRASILEIRA DE ZOOTECNIA, 36., 1999., Porto Alegre. Anais... Porte Alegre, Sociedade Brasileira de Zootecnia, 1999. p.354-357.

SILVA SOBRINHO A.G.; GASTALDI, K.A.; GARCIA, C.A. et al. Diferentes dietas e pesos ao abate na produção de órgão de cordeiros. Revista Brasileira de Zootecnia, v.32, n.6, supl. 1, p.1792-1799, 2003

TONETTO, C.J.; PIRES, C.C.; MÜLlER, L. et al. Ganho de peso e características de carcaça de cordeiros terminados em pastagem natural suplementada, pastagem cultivada de azevém (Leolium multiflorum Lam) e confinamento. Revista Brasileira de Zootecnia, v.33, n.1, p.225-233, 2004. 